Check for updates

The BMJ

Cite this as: BMJ 2020;371:m4347 http://dx.doi.org/10.1136/bmj.m4347 Published: 09 November 2020

\section{Covid-19: Vaccine candidate may be more than $90 \%$ effective, interim results indicate}

\author{
Elisabeth Mahase
}

A vaccine candidate developed by Pfizer and BioNTech may be more than $90 \%$ effective in preventing covid-19 in participants without prior infection, the first interim analysis of the phase III trial shows.

The research team evaluated the results after 94 cases of covid-19 had been confirmed. The study began in July and has so far enrolled nearly 44000 participants, just under 39000 of whom have received a second dose of the mRNA vaccine candidate (as of 8 November).

The team's announcement said, “The case split between vaccinated individuals and those who received the placebo indicates a vaccine efficacy rate above $90 \%$, at seven days after the second dose. This means that protection is achieved 28 days after the initiation of the vaccination, which consists of a two-dose schedule." However, the results, which are the first from any phase III trial of a covid-19 vaccine, have been shared only by press release and have not yet been published or peer reviewed.

Experts have welcomed the news but emphasised the importance of seeing the full results and understanding many of the details not yet made public, including the participants' demographic details and the severity of the cases reported in the trial.

The companies intend to submit the vaccine for emergency use authorisation by the US Food and Drug Administration after they have collected safety data for the two months after the second dose of the vaccine. They are expected to reach this milestone by the third week of November. They also "plan to submit data from the full phase II trial for scientific peer-review publication" but have not specified when this will happen.

The clinical trial will continue through to final analysis at 164 confirmed cases, so as to collect further data and assess other endpoints such as efficacy based on cases occurring 14 days after the second dose.

The researchers will also evaluate the potential for the vaccine to protect against the virus in people who have had prior exposure and to offer prevention against severe covid-19 disease. Participants will be monitored for long term protection and safety for an additional two years after their second dose.

Azra Ghani, chair of infectious disease epidemiology at Imperial College London, said, “These new results represent the first demonstration of substantial efficacy of a vaccine candidate against covid-19 disease, which is very welcome news. It is important to bear in mind that these are early results based on a relatively small number of cases. In addition, the efficacy estimate is based on seven days of follow-up of participants following the second dose; further data in the coming weeks and months will provide a better picture of longer term vaccine efficacy."

Eleanor Riley, professor of immunology and infectious disease at the University of Edinburgh, said, "At face value, this is exceptionally good news: a vaccine that is $90 \%$ effective at preventing symptomatic cases of covid-19 and with millions of doses available by the end of the year.

"However, the full dataset on which the claim is based has not yet been released, and so we don't know exactly what has been found. The two companies are at pains to point out that the trial participants are ethnically diverse, which is good, but say nothing about the age of people in the trial. If a vaccine is to reduce severe disease and death, and thus enable the population at large to return to their normal day to day lives, it will need to be effective in older and elderly members of our society. We also know nothing yet about the severity of cases that were seen in the trial, whether infection or infectiousness was prevented, or how long the immunity is expected to last. But, I think we have reason to be cautiously optimistic."

The companies have said around $42 \%$ of the trial's global participants and $30 \%$ of US participants have "racially and ethnically diverse backgrounds."

Michael Head, senior research fellow in global health at the University of Southampton, said, "If the final results show an effectiveness of anywhere near $90 \%$, with response in elderly and ethnic minority populations, that is an excellent result for a first generation vaccine."

He said that logistical problems with the vaccine would need to be tackled, as it has been reported that it requires storage at $-70^{\circ} \mathrm{C}$. "That is not necessarily routinely available in most health centres even in the UK, let alone globally.” 